\title{
FULL STRESS TENSOR DETERMINATION IN A TEXTURED AEROSPACE ALUMINIUM ALLOY PLATE USING SYNCHROTRON X-RAY DIFFRACTION
}

\author{
V. STELMUKH*, L. EDWARDS and S. GANGULY \\ Department of Materials Engineering, The Open University, \\ Walton Hall, Milton Keynes, MK7 6AA, UK
}

(Received 13 September 2003)

\begin{abstract}
Triaxial strain scanning has been performed on a $7 \mathrm{~mm}$ thick 7150 -aluminium alloy Variable Polarity Plasma Arc (VPPA) weld using synchrotron X-rays. It has been demonstrated that the use of specific advantageous $(h k l)$ peaks that occur in this highly textured aerospace alloy plate permits fast deep measurements in reflection as well as in transmission. The $\sin ^{2} \psi$ method was also utilised to monitor the variation in deviatoric stress component $\left(\sigma_{11}-\sigma_{33}\right)$ across the weld in both the test-piece and a comb like reference specimen used to map the changes in stress-free lattice spacings over the weld. The results suggest that microstresses have little effect on macrostress determination, particularly in the Heat Affected Zones (HAZ). Though small in magnitude, the macrostresses still present in the reference specimen can be allowed for to correct the stress tensor in the plate. Maximum tensile longitudinal stress values remain unchanged, whereas the stress condition in some parts of the HAZ is well described as biaxial after the proposed correction.
\end{abstract}

Keywords: Residual stress; Synchrotron diffraction; Texture; Aluminium variable polarity plasma arc weld

\section{INTRODUCTION}

Extensive research has been exploring a variety of advanced welding technologies (Mendez and Eagar, 2001) to reduce cost and provide innovative design options in airframe manufacturing. For damage tolerance of safety critical parts, it is vital that high quality joints of high strength precipitation hardened aluminium alloys currently considered for Very Large Aircraft (VLA) wing construction exhibit as much resistance to crack growth as possible. In particular, the behaviour of fatigue cracks in terms of their location, growth rates and direction of propagation must be related to the residual stresses present in the welded structures. In order to obtain this knowledge accurate reliable methods enabling determination of the residual stress profiles around candidate welds must be developed. In our recent work (Stelmukh et al., 2002)

\footnotetext{
*Corresponding author. Tel.: +44(0) 1908 655105. Fax +44(0) 1908653858.

E-mail: V.Stelmukh@open.ac.uk
} 
a hybrid neutron/synchrotron diffraction technique was designed to optimise the mapping of the full 3D stress tensor in a Metal Inert Gas (MIG) welded 7150W51(T6) aluminium alloy. The purpose of this contribution is to demonstrate that hard X-rays alone can be used for effective triaxial strain measurements in plates of this highly textured alloy, in the present case, welded using the Variable Polarity Plasma Arc (VPPA) process.

\section{EXPERIMENTAL}

Two $12.6 \mathrm{~mm}$ thick $500 \mathrm{~mm}$ long AA7150 plates were VPPA welded in the W51 condition (solution treatment, quenching followed by stress relief by stretching $(\sim 2 \%)$ in the rolling direction (RD)) using the so-called 'keyhole' mode (Cary, 1998), which provides for full-penetration single-pass welding. The weld orientation was parallel to the RD of the parent plates. A conventional peak aging treatment was applied to the specimen following welding, equivalent to T6, that resulted in a T651 condition in the far field parent material and related conditions in the HAZ and fusion zone. Finally, a $280 \mathrm{~mm}$ square test-piece was cut off and then reduced in thickness to $7 \mathrm{~mm}$ by machining from both sides in increments of $0.5 \mathrm{~mm}$ to simulate a likely aerospace manufacturing process.

Synchrotron X-ray diffraction has recently emerged as an exceptionally powerful technique for bulk strain scanning in Al-based alloys and MMCs (e.g. Withers et al., 2002). It has been traditionally believed that it is impractical to perform synchrotron through-thickness strain measurements on plate specimens in reflection (see e.g. Webster et al., 2001), as the X-ray path length is large $(=2 D / \sin \theta)$ for the usable diffraction angles $\left(\theta<12.5^{\circ}\right)$ and depths, $D$, exceeding $1 \mathrm{~mm}$. However, it is apparent that estimates of sufficient exposure times made for a texture-free material will not be valid for highly textured plates, where the most advantageous $(h k l)$ reflection can be selected for the strain measurement in the normal (or through-thickness) direction (ND). 7XXX alloy plates are typically characterised by a strong texture in which Brass, $\{011\}\langle 211\rangle$, is usually one of the main components. This component strengthens with depth as shown by a number of investigators (see e.g. Chang et al., 1998; Stelmukh and Edwards, 2002) and can be inferred from the through-thickness intensity variation for the (220) peak (see Fig. 1) taken from a previous neutron diffraction experiment (Stelmukh et al., 2002). Inverse pole figures obtained by the Electron BackScatter Diffraction (EBSD) from the middle of the present AA7150 plate are shown in Fig. 2, from which the presence of a much weaker Cube, $\{100\}\langle 001\rangle$, component can also be inferred.

Synchrotron X-ray diffraction measurements were carried out on the ID31 beam-line at the European Synchrotron Radiation Facility (ESRF) using the diffractometer recently transferred from BM16. Because of the large dimensions of the test-piece a special set-up was designed to operate the diffractometer in its strain-scanning mode using some components of the computer controlled positioning equipment developed by Webster and co-workers. Nevertheless, the measurement interval across the weld was still limited to the positions ranging from -25 to $+50 \mathrm{~mm}$ with respect to the weld centre-line. The experiment was conducted using $45 \mathrm{keV} \mathrm{X-rays.} \mathrm{Outside}$ the fusion zone, the measurements in reflection were found to be practically viable to the depths of $2.5 \mathrm{~mm}$ below the plate's surfaces (see Fig. 1), as individual count times 


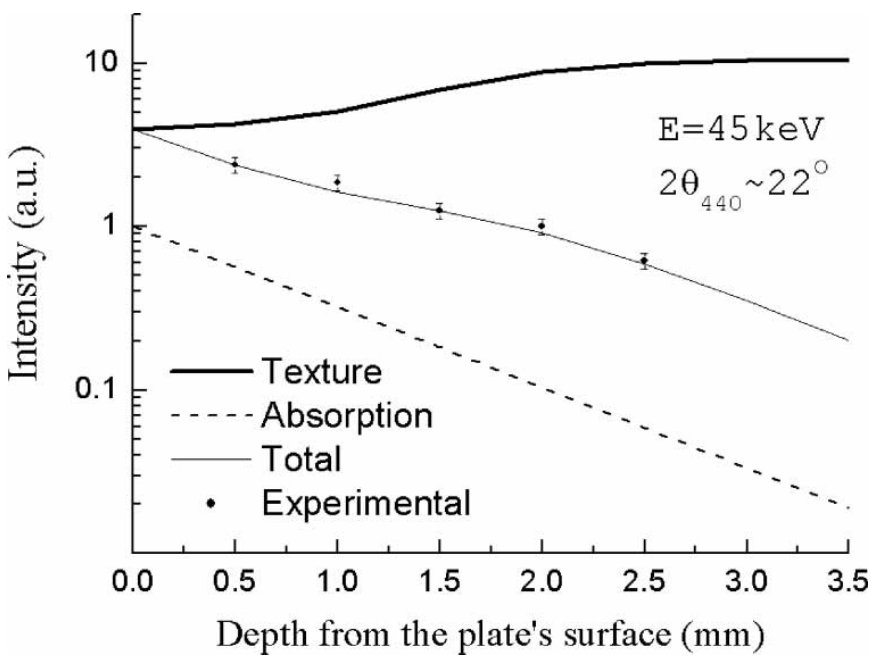

FIGURE 1 Through-thickness intensity variation of the (440) peak measured in reflection mode. Data represented by the bold line were obtained from a previous neutron diffraction experiment.

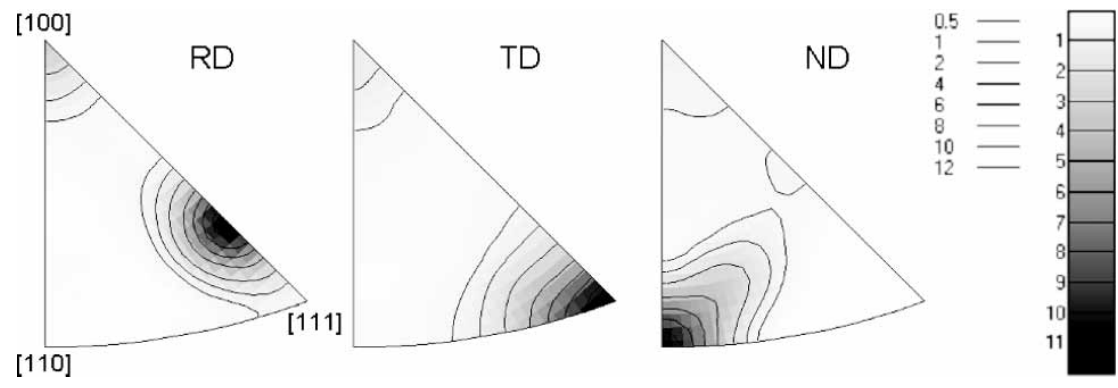

FIGURE 2 Inverse pole figures obtained for the middle of the parent plate using $10-\mu \mathrm{m}$ steps within a $1 \mathrm{~mm}$ square grid pattern. TD and ND stand for the transverse and normal direction respectively.

per point did not exceed $30 \mathrm{~s}$. The (440) peak was used to measure the ND strain $\left(\varepsilon_{33}\right)$, whereas the longitudinal $\left(\varepsilon_{11}\right)$ and transverse $\left(\varepsilon_{22}\right)$ strains were measured in transmission using the (422) and (222) peaks respectively.

Schematics of all the measurement geometries used are shown in Fig. 3. The dimensions of the diamond shaped sampling volume (gauge) were defined by the horizontal width of the incident beam $(w=2 \mathrm{~mm})$ and the incident and diffracted beam apertures, $v_{i}$ and $v_{d}$, both set to $0.5 \mathrm{~mm}$. The latter is practically equal to the shortest diagonal of the gauge, while its longest diagonal, $L$, is strongly dependent on the $(h k l)$ peak diffraction angle, $2 \theta$. The calculated values of $L$ for the peaks used are given in Fig. 3. The effective spatial resolution, however, is much smaller than $L$, as $75 \%$ of detected intensity is contributed by the central portion of the gauge.

The 'elastic stress free' reference measurements were made on a 'comb' specimen, $120 \mathrm{~mm}$ long, removed by Electro Discharge Machining from another weld prepared under nominally identical conditions. The dimensions of each individual 'tooth' of the comb were $3.0 \times 2.7 \mathrm{~mm}^{2}$ in the LD (Longitudinal Direction)-TD (Transverse 
(a)

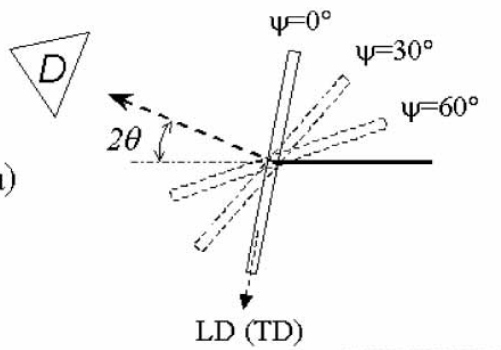

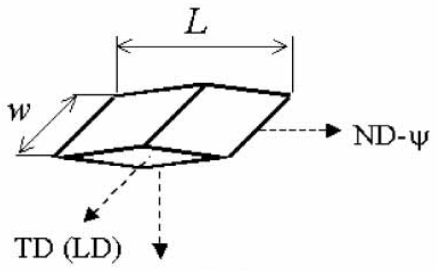

LD- $\psi(T D)$

\begin{tabular}{|c|c|c|c|}
\hline$(\mathrm{hkl})$ & $(222)$ & $(422)$ & $(440)$ \\
\hline $2 \theta(\mathrm{deg})$ & 13.5 & 19.1 & 22.1 \\
\hline$L(\mathrm{~mm})$ & 4.3 & 3.0 & 2.6 \\
\hline$(\mathrm{m})$ & &
\end{tabular}

(b)
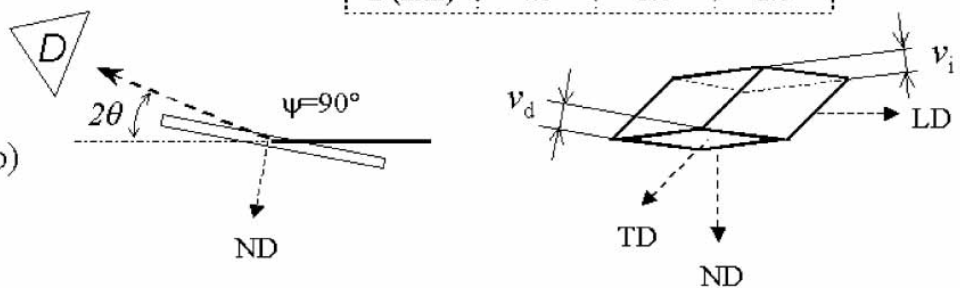

FIGURE 3 The experimental set-ups and corresponding orientations of the gauge volume for strain scanning in transmission (a) and reflection (b). LD stands the longitudinal direction. See the text for other details.

Direction) plane and $10.5 \mathrm{~mm}$ along ND. The allowance for a possible effect of the retained intergranular stresses on the macroscopic strain determination was made by measuring the reference specimen at orientations corresponding to equivalent measurements in the bulk material (see e.g. Krawitz and Winholtz, 1994). The measured strains, $\varepsilon_{i i}=\left(d_{h k l}-d_{h k l}^{\mathrm{ref}}\right) / d_{h k l}^{\mathrm{ref}}$, were then converted to stresses using the reasonable assumption that the measurement directions were aligned with the principal stress axes, and Hooke's law for isotropic solids:

$$
\sigma_{i i}=\frac{E}{1+v} \varepsilon_{i i}+\frac{v E}{(1+v)(1-2 v)}\left(\varepsilon_{11}+\varepsilon_{22}+\varepsilon_{33}\right)
$$

where the elastic modulus, $E$, was taken as $71 \mathrm{GPa}$ and Poisson's ratio, $v$, as 0.35 . The above formula and constants are found to be suitable for the stress calculations when comparing their results with those obtained using a generalised Hooke's law for an aluminium plate with the (011) [2111] ideal orientation (see e.g. Hauk, 1997).

The method of measuring the lattice strain in especially oriented groups of crystallites (see e.g. Hauk and Nikolin, 1988) was also used to monitor the variation in deviatoric stress component $\left(\sigma_{11}-\sigma_{33}\right)$ across the weld in both the test-piece and the reference specimen. Based on the results of texture examination presented above, the additional measurements were made at an orientation angle, $\psi$, of $30^{\circ}$ and $60^{\circ}$ (see Fig. 3a) using the (440) and (422) peaks respectively. Together with the data obtained at $\psi=0^{\circ}$ (for the LD strain measurement) and $\psi=90^{\circ}$ (for the ND strain measurement) they formed the basis for the $a v s \sin ^{2} \psi$ analysis, for which the values of the lattice parameter, $a$, were derived from the measured lattice spacings, $d_{422}$ and $d_{440}$. In 
order to calculate the deviatoric stress component; the $(h k l)$ dependent X-ray elastic constant, $s_{2}(h k l)$, for Al-based materials given in (Hauk, 1997) and the slope, $m$, found by the linear regression of the $a v s \sin ^{2} \psi$ distribution were used in the well-known formula (see e.g. Hauk, 1997):

$$
\sigma_{11}-\sigma_{33}=\frac{2 m}{a_{0} s_{2}}
$$

where an approximate value of the 'stress free' lattice parameter, $a_{0}$, can be replaced with the average lattice parameter observed in the experiment without sacrifice of precision. It is worth noting here that $s_{2}(422)$ is equal to $s_{2}(440)$, as the corresponding $[h k l]$ directions in cubic materials are characterized by the same elastic anisotropy factor (see e.g. Allen et al., 1985).

The synchrotron data for the reference specimen were complemented by the results of $\sin ^{2} \psi$ experiments performed using laboratory X-rays (CuK radiation) on a Brucker D5005 diffractometer. For the middle of each 'tooth' of the reference comb, the measurements were taken from its diamond polished TD-ND surface under the same conditions (i.e. orientations and reflections). As no measurements could be made at $\psi=90^{\circ}$, their number was increased by using pseudo-negative values of $\psi$, achieved via the $180^{\circ}$ rotation of the specimen (i.e. $\varphi$-rotation).

\section{RESULTS AND DISCUSSION}

Figure 4 shows the $d_{440}$ distributions obtained by the deepest measurements in reflection. Data for the fusion zone (which approximately covers $\pm 4 \mathrm{~mm}$ from the weld centre-line) are absent because the Brass texture component discontinues there. ND measurements from the through-thickness midline are possible but were not made, as they would require much longer counting times (see e.g. Fig. 1). Instead, the average of the shown distributions was used in the ND strain calculations since rather small through-thickness strain/stress gradients were found for all the measurement directions, as exemplified by the map of the longitudinal strain (see Fig. 5). This map was generated by Gsharp 3.2 software using bilinear interpolation of several hundred

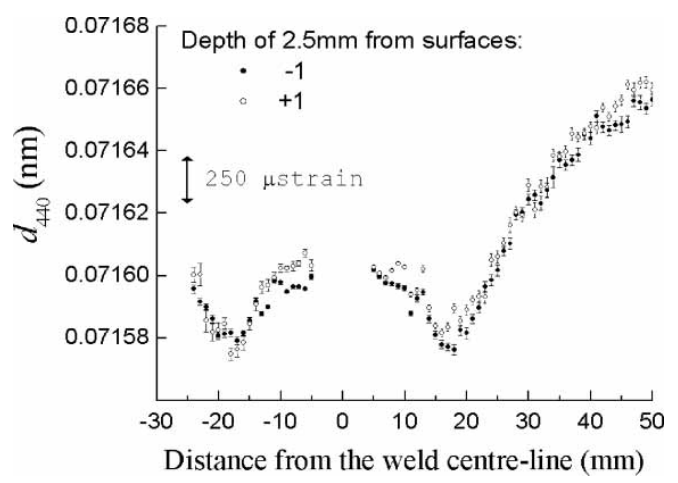

FIGURE 4 Data obtained in reflection from the textured regions outside the fusion zone. 


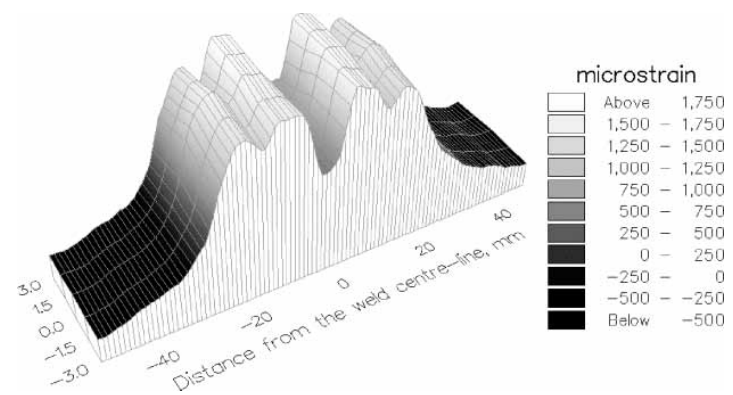

FIGURE 5 Map of the longitudinal strain $\left(\varepsilon_{11}\right)$ for the VPPA welded 7150-W51 (T6) aluminium alloy plate.
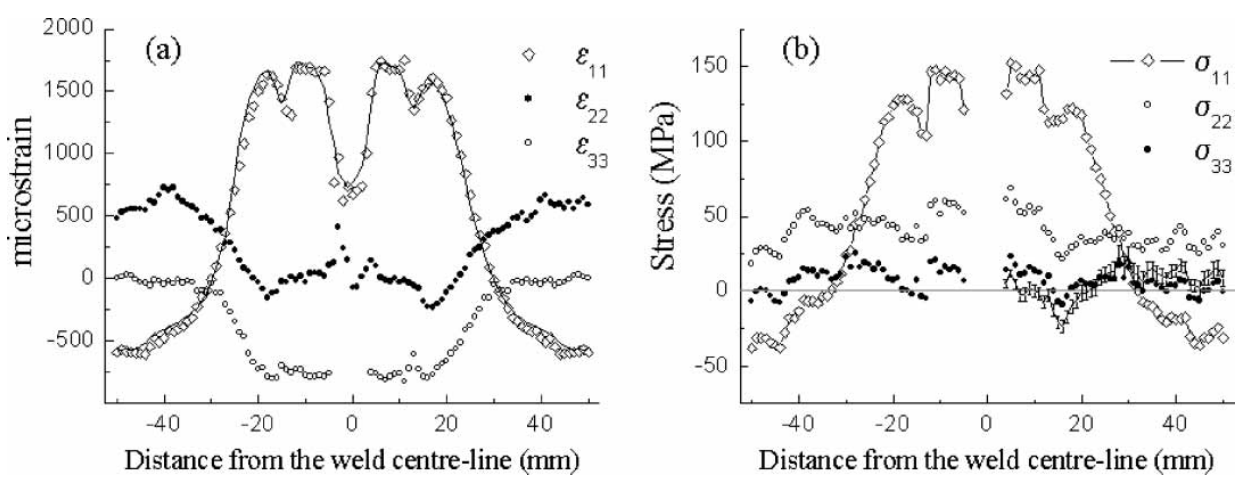

FIGURE 6 Midline strain (a) and stress (b) distributions. Error bars are not given, as their size is smaller than the symbols used. The solid line in (a) represents the data used to plot the map shown in Fig. 5, whilst the solid line with error bars in (b) is the 'near-zero' variation of the corrected $\sigma_{33}$ (for the positive side of the weld centre-line).

individual strain values. Longitudinal tension is characterised by the presence of two distinct maxima in the HAZ (at about \pm 8 and $\pm 18 \mathrm{~mm}$ from the weld centre-line) separated by a deep minimum in the fusion zone. Triaxial strain data obtained for the midline are shown in Fig. 6(a). A noticeable scatter in the values of $\varepsilon_{22}$ in the fusion zone is attributable to the reduced number of grains in the gauge volume and related difficulties with texture average when using the (222) reflection characterised by relatively small multiplicity factor $\left(P_{222}=8\right.$, whereas $\left.P_{422}=24\right)$.

The calculated stress distributions are shown in Fig. 6(b). The typical error in individual stress values, calculated using uncertainties of the peak positions for both the test-piece and the reference specimen, is about $2 \mathrm{MPa}$. The longitudinal stress profile largely inherits all the features of the corresponding strain distribution. At the weld centre-line the value of stress is estimated to be about $40 \mathrm{MPa}$. This can be done using the reasonable assumption that the behaviour of $\varepsilon_{33}$ in the fusion zone is similar to that of $\varepsilon_{22}$. The maximum tensile longitudinal stresses, ranging from 140 to $150 \mathrm{MPa}$, are about $25 \%$ of the yield strength of AA7150-T651 alloy, which is $570 \mathrm{MPa}$ at room temperature (Lin et al., 2003). Starting from about $\pm 22 \mathrm{~mm}$, this stress declines rapidly with a distance from the weld centre-line, switching from tension to compression at $32-34 \mathrm{~mm}$. This is near the outer boundaries of the HAZ $( \pm 37 \mathrm{~mm})$, which were found by measuring hardness. The transverse stress is comparatively small and 
tensile at all locations. The latter is probably predetermined by the existing stress condition of the rolled plates (see e.g. Prime and Hill, 2002) before welding.

Typical $a v s \sin ^{2} \psi$ distributions obtained are shown in Fig. 7. For the HAZ in the test-piece, a straight line fits the four points rather well, which is reflected in the small stress error (about $2 \mathrm{MPa}$ ). The diffraction peak widths vary significantly. In the regions of the HAZ bordering the fusion zone, the Full Width at Half Maximum (FWHM) of the (422) peak approaches that of Al powder (see Fig. 8), indicating low, if any, plastic deformation. As can be seen from Fig. 9(a), where the deviatoric stress is plotted for each measurement position within one side of the weld centre-line, nonlinearity of the $a v s \sin ^{2} \psi$ data noticeably increases when approaching the parent metal. This can be regarded as a marker of the increased influence of intergranular stresses, which often give rise to the non-linearities (see e.g. Hauk, 1997). Based on these observations, one can anticipate that microstresses will have little effect on the macrostress determination in high tensile areas, which usually are of considerable importance for the damage tolerance analysis.

The results of the $\sin ^{2} \psi$ analysis for the reference comb (see Fig. 9c) suggest that macrostresses are still present in the reference specimen. They are likely to be small
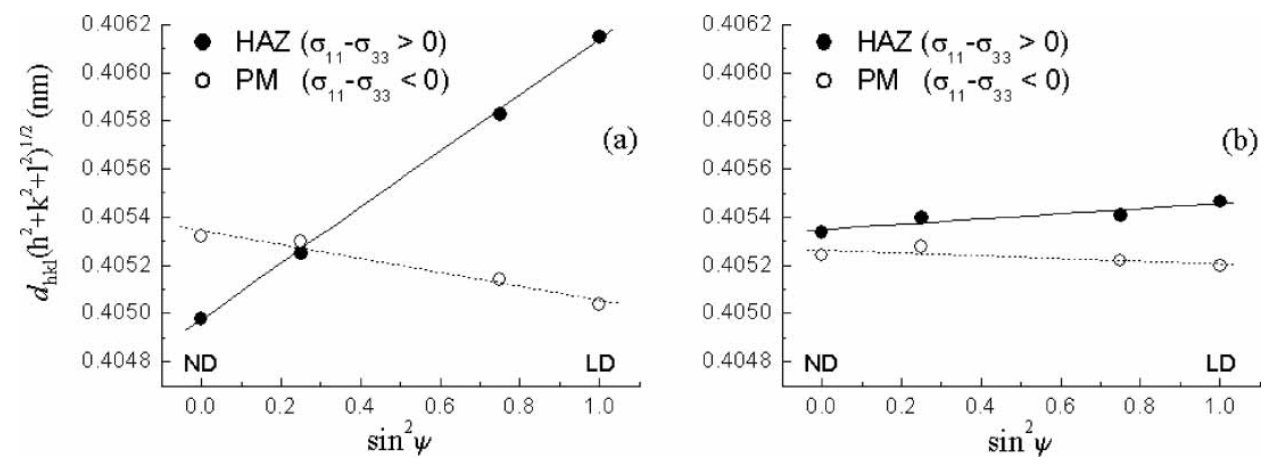

FIGURE 7 Typical $a v s \sin ^{2} \psi$ distributions obtained for the test piece (a) and the reference (b) specimen using synchrotron $\mathrm{X}$-ray radiation.

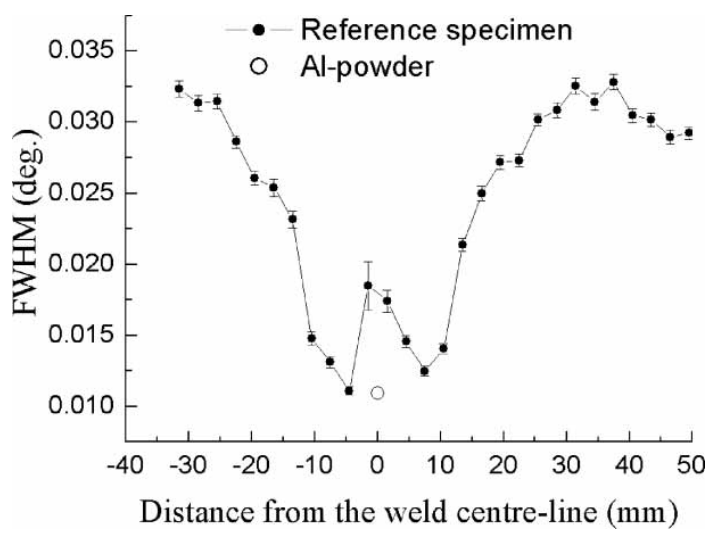

FIGURE 8 The (422) peak width distribution. 


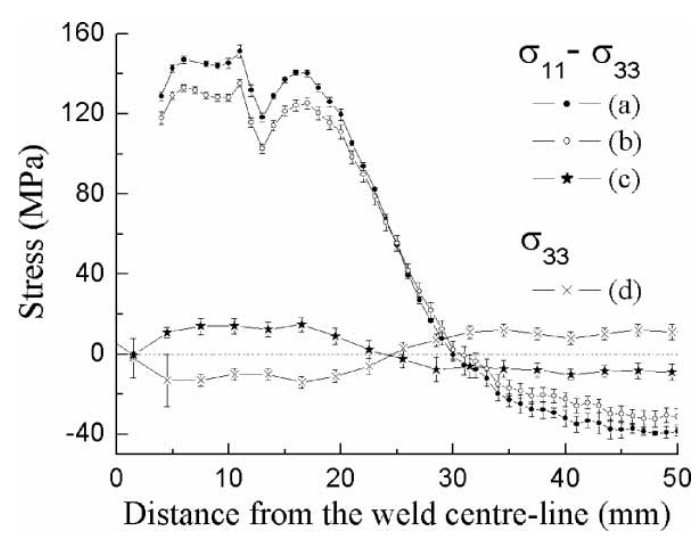

FIGURE 9 Midline stress distributions for the weld (a, b) and reference comb (c, d) determined by the $\sin ^{2} \psi$ method (a, c, d) and from triaxial stress measurements (b).

in magnitude, which is supported by the results of similar measurements made using laboratory X-rays (Fig. 9d), where boundary condition, $\sigma_{11}=0$, holds. On the assumption that the uniaxial approximation for the stress condition $\left(\sigma_{11}=\sigma_{22}=0\right.$ and $\sigma_{33} \approx$ $\pm 15 \mathrm{MPa}$ ) in each 'tooth' of the reference specimen is valid, the stress tensor found from the triaxial strain measurements can be corrected to eliminate the apparent disagreement between the $\sigma_{11}-\sigma_{33}$ distributions shown in Fig. 9(a) and (b). When applied, this correction results in a biaxial stress condition $\left(\sigma_{33}=0\right)$ for the region in the HAZ corresponding to the first maximum of the longitudinal stress distribution (see the solid line with error bars in Fig. 6(b)). The highest tensile stress values, however, remain unchanged after this correction.

\section{CONCLUSION}

It has been shown that the full stress tensor can be determined in a textured aerospace alloy plate using synchrotron X-ray diffraction and the crystallite group method. The technique has been demonstrated on a large coupon of a 7150 VPPA weld. The adopted experimental strategy allowed fast residual strain measurements and the application of the $\sin ^{2} \psi$ method to many measurement positions in the test-piece and reference specimen. The affects of plastic anisotropy have been found to be small and the biaxial stress condition has also been shown to be an acceptable approximation for this combination of the welding process and post-welding thermo-mechanical treatment.

\section{Acknowledgments}

The authors wish to thank Dr. A. N. Fitch (beam-line responsible) of ESRF and Dr. J.R. Santisteban of the Open University for their continued support and assistance. We would also like to thank Peter Ledgard for his invaluable help in the preparation of the experiment. We express our gratitude to the Cranfield University Weld Engineering Research Centre for providing the 7150 VPPA weld, to Airbus (UK) and the UK's 
Engineering and Physical Sciences Research Council for their support via the grant "Weld processing design \& durability of welded aircraft assemblies".

\section{References}

Allen, A.J., Hutchings, M.T., Windsor, C.G. and Andreani, C. (1985). Neutron diffraction methods for the study of residual stress fields. Advances in Physics, 34, 445-473.

Cary, H.B. (1998). Modern Welding Technology, 4th Edn. Prentice-Hall.

Chang, S.C., Jiang, Q.D., Hu, J.R. and Chen, F.R. (1998). The texture and formation of shear bands in a hot rolled 7050 aluminium alloy. Scripta Mater., 39, 583-588.

Hauk, V. and Nikolin, H.-J. (1988). The evaluation of the distribution of residual stresses of the I. kind (RS I) and of the II. kind (RS II) in textured materials. Textures and Microstructures, 8\&9, 693-716.

Hauk, V. (1997). Structural and Residual Stress Analysis by Nondestructive Methods. Elsevier, Amsterdam.

Krawitz, A.D. and Winholtz, R.A. (1994). Use of position-dependent stress-free standard for diffraction stress measurements. Mater. Sci. Eng. A, 185, 123-130.

Lin, J., Stelmukh, V., Ganguly, S., Edwards, L. and Irving, P.E. (2003). The effects of residual stress and HAZ on fatigue crack growth in MIG welded 2024 and 7150 Aluminium. To be published in Fatigue and Fracture of Engineering Materials and Structures.

Mendez, P.F. and Eagar, T.W. (2001). Welding processes for aeronautics. Advanced Materials and Processes, 5, 39-43.

Prime, M.B. and Hill, M.R. (2002). Residual stress, stress relief, and inhomogeneity in aluminium plate. Scripta Mater., 46, 77-82.

Stelmukh, V., Edwards, L., Santisteban, J.R., Ganguly, S. and Fitzpatrick, M.E. (2002). Weld stress mapping using neutron and synchrotron X-ray diffraction. Materials Science Forum, 404 407, 599-604.

Stelmukh, V. and Edwards, L. (2002). Optimizing neutron strain scanning by the use of electron backscatter diffraction. Microscopy and Analysis, 91, 15-16.

Webster, P.J., Oosterkamp, L.D., Browne, P.A., Hughes, D.J., Kang W.P., Withers, P.J., and Vaughan, P.M. (2001). Synchrotron X-ray residual strain scanning of a friction stir weld. J. Strain Anal. Eng., 36, 61-70.

Withers, P.J., Preuss, M., Webster, P.J., Hughes, D.J. and Korsunsky, A.M. (2002). Residual strain measurement by synchrotron diffraction. Materials Science Forum, 404-407, 1-12. 